\title{
An atypical case of hypomethylation at multiple imprinted loci
}

\author{
Emma L Baple ${ }^{\star, 1,3,5}$, Rebecca L Poole ${ }^{2,3,5}$, Sahar Mansour ${ }^{1}$, Catherine Willoughby ${ }^{1}$, I Karen Temple ${ }^{2,4}$, \\ Louise E Docherty ${ }^{2,3}$, Rohan Taylor ${ }^{1}$ and Deborah JG Mackay ${ }^{2,3}$
}

\begin{abstract}
Angelman syndrome (AS) and Prader-Willi syndrome (PWS) are caused by genetic and epigenetic mutations of the imprinted gene cluster on chromosome $15 q 13$. Although the imprinting mutations causing PWS and AS are essentially opposite in nature, remarkably, a small number of patients have been reported with clinical features of PWS but epigenetic mutations consistent with AS. We report here a patient who presented with clinical features partially consistent with both PWS and Beckwith-Wiedemann syndrome (BWS). Epimutations were found at both the AS/PWS and BWS loci, and additionally at the H19, PEG3, NESPAS and GNAS loci. This patient is therefore the first described case with a primary epimutation consistent with AS accompanied by hypomethylation of other imprinted loci.
\end{abstract}

European Journal of Human Genetics (2011) 19, 360-362; doi:10.1038/ejhg.2010.218; published online 5 January 2011

Keywords: Angelman; Prader-Willi; Beckwith-Wiedemann; imprinting; hypomethylation

\section{INTRODUCTION}

Imprinted genes are limited in expression potential by their parent of origin, which often coincides with parental-allele-specific DNA methylation. Imprinting disorders typically have heterogeneous clinical presentations. Some features, such as prenatal overgrowth, macroglossia or truncal obesity, are associated with more than one imprinting disorder, suggesting that imprinted genes control many and overlapping facets of human development. Moreover, in recent years, patients have been identified with imprinting disorders affecting more than one imprinted locus, and their clinical features may diverge from the 'classical' presentation of the disorder, possibly reflecting the complement of genes affected. ${ }^{1}$

Angelman syndrome (AS) and Prader-willi syndrome (PWS) are distinct genetic conditions caused by abnormalities at the 15q11-13 imprinted region. Patients with classical AS present with severe mental retardation, speech impairment, ataxia and seizures. They have a characteristic behaviour pattern of hyperactivity and a happy personality and episodes of inappropriate bursts of laughter. Dysmorphic features include microcephaly, macrostomia and prominence of the jaw. ${ }^{2}$ PWS is characterised by global developmental delay, hypotonia and feeding difficulties with failure to thrive in infancy. There is rapid weight gain usually between the ages of 1 and 4 years. This is due to hyperphagia, and results in truncal obesity. Patients have short stature and small hands and feet, and males have hypogonadism. The face is characterised by almond shaped eyes and an upward slant of the palpebral fissures. Temper tantrums and a tendency to an obsessive compulsive phenotype are commonly seen. ${ }^{3}$

AS is most commonly $(\sim 70 \%)$ caused by interstitial deletion of the maternally derived chr15q11-13. Other causes include paternal uniparental disomy (UPD; $1-2 \%)$, imprinting defect $(3-5 \%)$ and mutations in UBE3A (5-10\%). PWS is caused by a paternal deletion of the same region $(\sim 70 \%)$ or maternal UPD $(\sim 29 \%)$. A primary imprinting abnormality is rare. ${ }^{4}$

There are a small number of reported cases of patients with clinical features of PWS but a molecular diagnosis of AS..$^{5-7}$ In the majority of these cases partial loss of maternal methylation is observed.

We report here a 3-year-old girl, who presented with features of both PWS and a second imprinting disorder, Beckwith-Wiedemann syndrome (BWS). It was hypothesised that a methylation anomaly at more than one locus might explain the phenotype. Therefore, imprint status was investigated at both the PWS/AS and BWS regions, and thereafter at other imprinted loci.

\section{CASE REPORT}

The patient is one of four children born to unrelated Sri Lankan parents. Conception was natural and the pregnancy uncomplicated. Her birth weight at term was $3640 \mathrm{~g}$ (55th centile). A large, protruding tongue was noted in the neonatal period, the umbilicus was normal. There were no feeding problems, normal tone and no history of hyper- or hypoglycaemic episodes. Parathyroid, calcium and phosphate levels were normal. There was no history of organomegaly. Motor development was normal and she walked independently at 14 months. She was a shy child with some developmental delay particularly with speech, having no words by 2 years. She had excessive weight gain in her second year, and by 3.5 years her OFC was $53.3 \mathrm{~cm}$ (98th centile), height $96.9 \mathrm{~cm}$ (25th-50th centile) and weight $19.5 \mathrm{~kg}$ (95th centile). At the age of $3 \frac{1 / 2}{2}$ years, she has remained seizure free and is not ataxic. In addition to her large tongue, she has a round face and bilateral single palmar creases. She has two words and points to indicate her needs. In light of her macroglossia, BWS was suspected;

\footnotetext{
1SW Thames Regional Genetics Service, St George's NHS Trust, London, UK; ²Division of Human Genetics, University of Southampton School of Medicine, Southampton, UK; ${ }^{3}$ Wessex Regional Genetics Laboratory, Salisbury District Hospital, Salisbury, Wiltshire, UK; ${ }^{4}$ Wessex Clinical Genetic Service, The Princess Anne Hospital, Southampton University Hospitals Trust, Southampton, UK

*Correspondence: Dr EL Baple, Genetics Research Centre, Division of Biomedical Sciences, St George's University of London, Cranmer Terrace, London, SW17 ORE, UK.

E-mail: ebaple@sgul.ac.uk

5These authors contributed equally to this work.

Received 19 August 2010; revised 12 November 2010; accepted 16 November 2010; published online 5 January 2011
} 
however, because of the developmental delay and increased BMI, PWS also seemed a possibility.

\section{MATERIALS AND METHODS}

Ethical permission for this study was obtained from the Southampton and South West Hampshire Research ethics committee, REC reference number: 07/H0502/85.

DNA was extracted from peripheral blood by standard procedures.

Initially methylation was determined at the PWS/AS locus by methylationspecific PCR (MS-PCR) according to Kubuta $e t a l,{ }^{8}$ and by methylation-specific MLPA (MS-MLPA) at 11p15 using MRC Holland BWS kit ME030-B1 (MRC Holland bv, Amsterdam, The Netherlands) according to the manufacturer's instructions (as described by Scott et $a l^{9}$ ). KCNQ1OT1 and H19 results were confirmed by methylation-sensitive high-resolution melt (MS-HRM) analysis according to Alders et al. ${ }^{10}$

Subsequently, MS-PCR analysis of the patient and parental samples was carried out at the ARHI, PLAGL1, MEST1, GRB10, KCNQ1OT1, H19,
IGF2PO, DLK1, SNRPN, PEG3, GNAS, NESP55 and NESPAS loci as described previously. ${ }^{1,11}$ Pyrosequencing of SNRPN, GNAS, NESPAS and PEG3 was performed as previously described. ${ }^{1,12}$

Microsatellite analysis around the SNRPN, KCNQ1, H19 and PEG3 regions was performed using published microsatellites by standard methods. The markers investigated were: D15S817, D15S128, D15S1506, D15S122, D11S4177, D11S922, D11S4046, D11STH2, D11S1318, D11S4088, D11S4146, D19S210 and D19S887.

ZFP57 sequencing was performed as previously described. ${ }^{1}$

\section{RESULTS}

Initial testing of the SNRPN DMR by MS-PCR and the $11 \mathrm{p} 15.5$ region using MS-MLPA revealed a reduced intensity of the methylated maternal band at the SNRPN DMR, partial hypomethylation at $K v D M R$ and subtle hypomethylation at H19. The results at KvDMR and H19 were confirmed by MS-HRM (data not shown).

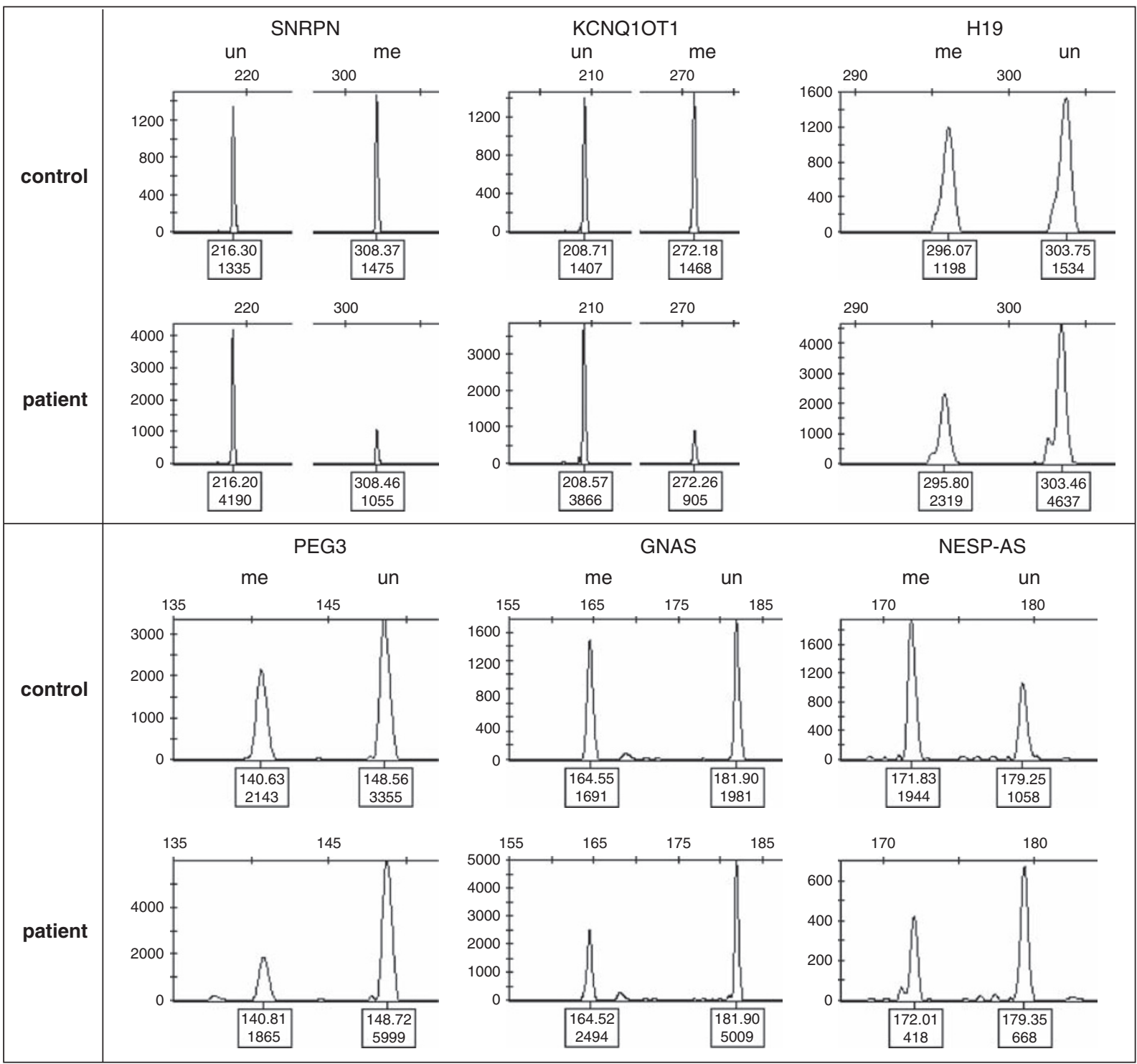

Figure 1 Electropherograms of methylation-specific PCR amplicons. The successive panels represent analysis of the SNRPN, KCNQ1OT1, H19, PEG3, GNAS and NESPAS DMRs, respectively. In each panel, the top trace illustrates a normal control, and the lower trace represents patient. Bars labelled 'me' and 'un' indicate 'methylated' and 'unmethylated' peaks, respectively. Hypomethylation in the patient is indicated by a reduction in the methylated peak height relative to the unmethylated peak when compared with the control. 
To extend these findings, MS-PCR was carried out at 13 imprinted loci across the genome. In addition to confirming the abnormalities at $11 \mathrm{p} 15$ and $15 \mathrm{q} 13$, partial loss of maternal methylation was seen at GNAS, NESPAS and PEG3 (Figure 1). The SNRPN epimutation and the additional epimutations at GNAS, NESPAS and PEG3 were confirmed by pyrosequencing.

Methylation analysis of the parental samples revealed normal methylation levels at all the loci investigated.

Microsatellite analysis of the patient and her parents around the regions of methylation abnormality showed biparental inheritance, excluding multiple regions of uniparental disomy as a cause of the imprinting anomalies. Sequencing of the ZFP57 gene revealed no mutations (data not shown).

\section{DISCUSSION}

We describe here a patient with clinical features suggestive of both PWS and BWS, who was found to exhibit hypomethylation of multiple imprinted loci (HIL).

The clinical features of this patient were remarkably mild given the extent of her methylation abnormalities. Macroglossia and single palmar creases are both diagnostic features of BWS, and consistent with the imprinting anomaly observed. However, the normal birth weight, umbilicus, glycaemia and lack of hemihypertrophy were inconsistent with BWS. The patient's high BMI and developmental delay were consistent with the initial clinical suspicion of PWS, though normal tone and normal facial appearance were inconsistent with PWS. Moreover, she had no ataxia, microcephaly or seizures to suggest AS. We suggest that this case is an example of the clinical picture that may emerge when hypomethylation affects multiple imprinted loci.

Methylation abnormalities at multiple imprinted loci have been described previously in other imprinting syndromes including transient neonatal diabetes (TND), ${ }^{1,13}$ BWS $^{14-16}$ and Russell-Silver syndrome ${ }^{17,18}$. The only gene mutation so far associated with such disorders is mutation of $Z F P 57$, which was found in $\sim 50 \%$ of patients with TND HIL. ${ }^{1}$ This patient showed no evidence of a ZFP57 mutation, and her pattern of methylation abnormalities seems distinct from that seen in either TND HIL or BWS HIL. Interestingly, the epimutations observed include mosaic hypomethylation of the maternally methylated SNRPN, KCNQ1OT1, PEG3 and NESPAS loci, and the paternally methylated $\mathrm{H} 19$ locus. This pattern of abnormalities suggests failure of imprint maintenance, rather than its establishment.

It would be interesting to study further cases with an epimutation consistent with AS but presenting with features of PWS, as it is likely that some of these cases will also harbour additional methylation changes.

This case is a significant addition to the weight of evidence suggesting that patients with HIL may go undiagnosed because their presentation may blur the boundaries of recognised imprinting disorders. We, therefore, suggest that epigenetic analysis at multiple loci should be carried out in patients presenting with clinical signs that could be consistent with more than one of the classical imprinting disorders. It is becoming clear that the clinical picture in patients with HIL is extremely variable and that epigenetic as well as genetic mutations should be suspected in any patient with an unusual syndromic presentation.

\section{CONFLICT OF INTEREST}

The authors declare no conflict of interest.

\section{ACKNOWLEDGEMENTS}

We thank the family for supplying blood samples for scientific research. A part of this work was supported by the Newlife foundation (formerly BDF).

1 Mackay DJ, Callaway JL, Marks SM et al: Hypomethylation of multiple imprinted loci in individuals with transient neonatal diabetes is associated with mutations in ZFP57. Nat Genet 2008; 40: 949-951.

2 Buggenhout GV, Fryns J-P: Angelman syndrome. Eur J Hum Genet 2009; 17: 1367-1373.

3 Cassidy SB, Driscoll DJ: Prader-Willi syndrome. Eur J Hum Genet 2009; 17: 3-13.

4 Horsthemke B, Wagstaff J: Mechanisms of imprinting of the Prader-Willi/Angelman region. Am J Med Genet A 2008; 146A: 2041-2052.

5 Gillessen-Kaesbach G, Demuth S, Thiele H, Theile U, Lich C, Horsthemke B: A previously unrecognised phenotype characterised by obesity, muscular hypotonia and ability to speak in patients with Angelman syndrome caused by an imprinting defect. Eur J Hum Genet 1999; 8: 241.

6 De Molfetta GA, Felix TM, Riegel M, Ferraz VE, de Pina Neto JM: A further case of a Prader-Willi syndrome phenotype in a patient with Angelman syndrome molecular defect. Arq Neuropsiquiatr 2002; 60: 1011-1014.

7 Camprubí C, Coll MD, Villatoro S et al: Imprinting center analysis in Prader-Willi and Angelman syndrome patients with typical and atypical phenotypes. Eur J Med Genet 2007; 50: 11-20.

8 Kubota T, Das S, Christian SL et al: Methylation-specific PCR simplifies imprinting analysis. Nat Genet 1997; 16: 16-17.

9 Scott RH, Douglas J, Baskcomb L et al: Methylation-specific multiplex ligationdependent probe amplification (MS-MLPA) robustly detects and distinguishes $11 \mathrm{p} 15$ abnormalities associated with overgrowth and growth retardation. J Med Genet 2008; 45: 106-113.

10 Alders M, Bliek J, vd Lip K, vd Bogaard R, Mannens M: Determination of KCNQ10T1 and $\mathrm{H} 19$ methylation levels in BWS and SRS patients using methylation-sensitive high-resolution melting analysis. Eur J Hum Genet 2009; 17: 467.

11 Poole RL, Baple E, Crolla JA, Temple IK, Mackay DJ: Investigation of 90 patients referred for molecular cytogenetic analysis using aCGH uncovers previously unsuspected anomalies of imprinting. Am J Med Genet A 2010; 152A: 1990-1993.

12 White HE, Durston VJ, Harvey JF, Cross NC: Quantitative analysis of SNRPN gene methylation by pyrosequencing as a diagnostic test for Prader-Willi syndrome and Angelman syndrome. Clin Chem 2006; 52: 1005-1013.

13 Mackay DJ, Boonen SE, Clayton-Smith J et al: A maternal hypomethylation syndrome presenting as transient neonatal diabetes mellitus. Hum Genet 2006; 120: 262-269.

14 Rossignol S, Steunou V, Chalas C et al: The epigenetic imprinting defect of patients with Beckwith-Wiedemann syndrome born after assisted reproductive technology is not restricted to the 11 p15 region. J Med Genet 2006; 43: 902-907.

15 Bliek J, Alders M, Maas SM et al: Lessons from BWS twins: complex maternal and paternal hypomethylation and a common source of haematopoietic stem cells. Eur J Hum Genet 2009; 17: 1625-1634.

16 Bliek J, Verde G, Callaway J et al: Hypomethylation at multiple maternally methylated imprinted regions including PLAGL1 and GNAS loci in Beckwith-Wiedemann syndrome. Eur J Hum Genet 2009; 17: 611-619.

17 Azzi S, Rossignol S, Steunou V et al: Multilocus methylation analysis in a large cohort of 11p15-related foetal growth disorders (Russell Silver and Beckwith Wiedemann syndromes) reveals simultaneous loss of methylation at paternal and maternal imprinted loci. Hum Mol Genet 2009; 18: 4724-4733.

18 Turner CLS, Mackay DJ, Callaway JA et al: Methylation analysis of 79 patients with growth restriction reveals novel patterns of methylation change at imprinted loci. Eur J Hum Genet 2010; 18: 648-655. 